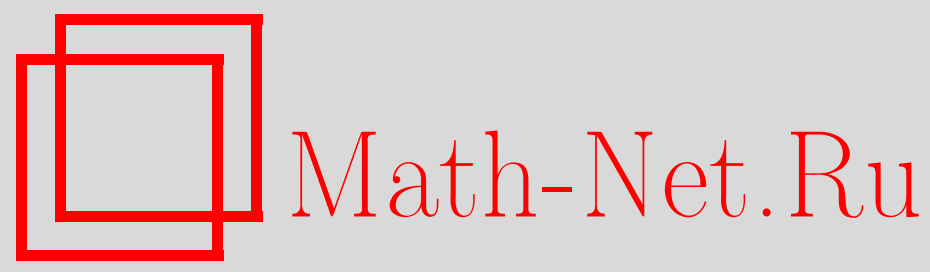

А. В. Покровский, Теоремы о среднем для решений линейных дифференциальных уравнений с частными производными, Матем. заметки, 1998, том 64, выпуск 2, 260-272

DOI: https://doi.org/10.4213/mzm1394

Использование Общероссийского математического портала Math-Net.Ru подразумевает, что вы прочитали и согласны с пользовательским соглашением http://www.mathnet.ru/rus/agreement

Параметры загрузки:

IP : 54.197 .130 .99

26 апреля 2023 г., $12: 36: 24$






\section{ТЕОРЕМЫ О СРЕДНЕМ ДЛЯ РЕШЕНИЙ ЛИНЕЙНЫХ ДИФФЕРЕНЦИАЛЬНЫХ УРАВНЕНИЙ С ЧАСТНЫМИ ПРОИЗВОДНЫМИ}

\section{А. В. Покровский}

Рассматриваются теоремы типа теорем о среднем для решений линейных дифференциальных уравнений с постоянными коэффициентами и нулевой правой частью, удовлетворяющих следующему условию однородности относительно заданного вектора $\boldsymbol{M}$ с натуральными компонентами: для каждой частной производной, входящей в рассматриваемое уравнение, скалярное произведение вектора, составленного из порядков этой производной по каждому переменному, на вектор $\boldsymbol{M}$ не зависит от этой производной. Основным результатом работы является обобщение известной теоремы Зальцмана. Приводятся следствия.

Библиографоя: 11 названий.

Введение. Пусть $\mu$ - комплексная борелевская мера с носителем на шаре $B:=$ $\left\{\boldsymbol{x} \in \mathbb{R}^{n}:|\boldsymbol{x}| \leqslant 1\right\}, n \geqslant 1, F_{\mu}$ - функционал, порожденный мерой $\mu$ в пространстве распределений $\mathscr{D}^{\prime}\left(\mathbb{R}^{n}\right)$ :

$$
F_{\mu}(\varphi)=\int \varphi(\boldsymbol{t}) d \mu(\boldsymbol{t})
$$

для каждой функции $\varphi \in C_{0}^{\infty}\left(\mathbb{R}^{n}\right)$. В работе Л. Зальцмана [1] рассматривались функции $f$, непрерывные в некоторой области $G \subset \mathbb{R}^{n}$, удовлетворяющие равенству

$$
\int f(\boldsymbol{x}+r \boldsymbol{t}) d \mu(\boldsymbol{t})=0
$$

при всех $\boldsymbol{x} \in G$ и $r>0$ таких, что $\{\boldsymbol{x}+\boldsymbol{r} \boldsymbol{t}: \boldsymbol{t} \in B\} \subset G$. Зальцман показал, что множество всех таких функций $f$ совпадает с множеством всех непрерьвньх слабых решений в $G$ некоторой системы, состоящей из конечного числа линейных дифференциальных уравнений с постоянными коэффициентами, зависящих только от меры $\mu$. Для сведения этой системы к одному дифференциальному уравнению $P(D) f=0$ необходимо и достаточно [1], чтобы оператор $P(D)$ был однородньг (т.е. содержал частные производные только одного порядка), а функционал $F_{\mu}$ имел вид $F_{\mu}=P(D) T$, где $T$ - некоторое распределение с носителем на $B, \widehat{T}(\mathbf{0}) \neq 0(\widehat{T}$ - преобразование Фурье-Лапласа

Работа выполнена при финансовой поддержке Российского фонда фундаментальных исследований, грант № 96-01-01366. 
распределения $T$ ). Этот результат Зальцмана представляет собой весьма общую теорему типа теорем о среднем для решений однородных дифференциальных уравнений с постоянньми коэффициентами. С другой стороны, были известны конкретные теоремы о среднем для решений уравнения теплопроводности, неоднородного в указанном выше смысле (см. [2], [3]). В настоящей работе мы, следуя подходу Зальцмана, распространим его результат на более широкий класс дифференциальных уравнений с постоянными коэффициентами, который, в частности, содержит оператор теплопроводности. Отметим, что мы имеем в виду приложения полученных результатов к исследованию некоторых функциональных классов, возникаюших в теории особых точек решений линейных дифоференциальных уравнений с частными производными, и планируем посвятить этому отдельную работу.

1. Определения и предварительные сведения. Прежде всего подчеркнем, что на протяжении всей работы нами рассматриваются только дифференциальные уравнения с постоянными коэффициентами и нулевой правой частью, и, говоря об однородности такого уравнения, мы имеем в виду, что дифференциальный оператор, стоящий в левой части, однороден, т.е. содержит частные производные только одного порядка.

Пусть $G$ - область в $\mathbb{R}^{n}, n \geqslant 1$. Как обычно, $C^{m}(G)$ - пространство всех комплекснозначных функций $\varphi$, имеющих непрерывные частные производные всех порядков $\leqslant m$ в $G, m \in \mathbb{N}_{0}:=\{0,1, \ldots\} ; C^{\infty}(G):=\bigcap_{m \geqslant 0} C^{m}(G), C(G):=C^{0}(G)$. Для замкнутой области $\bar{G}$ через $C^{m}(\bar{G}), m \in \mathbb{N}_{0}$, обозначим множество всех функций $f \in C^{m}(G)$, все частные производные которых порядка $\leqslant m$ допускают непрерывноепродолжениена $\bar{G}$; $C^{\infty}(\bar{G}):=\bigcap_{m \geqslant 0} C^{m}(\bar{G})$. Через $C_{0}^{\infty}(G)$ (соответственно $C_{0}^{\infty}(\bar{G})$ ) обозначим множество всех функций $\varphi \in C^{\infty}\left(\mathbb{R}^{n}\right)$, носитель которых $\operatorname{supp} \varphi$ есть компакт, принадлежащий области $G$ (замкнутой области $\bar{G}$ ). Как обычно, если $1 \leqslant p \leqslant \infty$, то $L_{p}(G)$ - линейное пространство всех функций $f$, интегрируемых по Лебегу в степени $p$ (существенно ограниченных при $p=\infty$ ) в области $G$, с обычной нормой, которая обозначается через $\|\cdot\|_{p, G}$. В случае $G=\mathbb{R}^{n}$ эта норма обозначается через $\|\cdot\|_{p}$.

Для произвольного мультииндекса $\boldsymbol{k}=\left(k_{1}, \ldots, k_{n}\right) \in \mathbb{N}_{0}^{n}$ положим

$$
\partial^{\boldsymbol{k}}=\left(\frac{\partial}{\partial x_{1}}\right)^{k_{1}} \cdots\left(\frac{\partial}{\partial x_{n}}\right)^{k_{n}}, \quad D^{\boldsymbol{k}}=(-i)^{|\boldsymbol{k}|} \partial^{\boldsymbol{k}}, \quad|\boldsymbol{k}|=k_{1}+\cdots+k_{n}
$$

Пусть $\boldsymbol{M}=\left(M_{1}, \ldots, M_{n}\right)$ - вектор с натуральньми компонентами, $|\boldsymbol{M}|=M_{1}+\cdots$ $+M_{n}$. Сопоставим каждому полиному $P(z), z=\left(z_{1}, \ldots, z_{n}\right) \in \mathbb{C}^{n}$, дифференциальньй оператор $P(D)$, полученньй заменой $z_{k}$ на

$$
\frac{1}{i} \cdot \frac{\partial}{\partial x_{k}}
$$

при каждом $k=1, \ldots, n$. Полином $P(\boldsymbol{z})$ (оператор $P(D)$ ) назьвается $\boldsymbol{M}$-однородныл $\boldsymbol{M}$, если существует $l \in \mathbb{N}_{0}$ такое, что $P(\boldsymbol{z}) \equiv \sum a_{\boldsymbol{k}} z^{\boldsymbol{k}}$, где сумма берется по множеству всех мультииндексов $\boldsymbol{k}=\left(k_{1}, \ldots, k_{n}\right) \in \mathbb{N}_{0}^{n}$ таких, что $|\boldsymbol{k} \boldsymbol{M}|:=k_{1} M_{1}+\cdots+k_{n} M_{n}=l$, $z^{\boldsymbol{k}}:=z_{1}^{k_{1}} \cdots z_{n}^{k_{n}}$. Для любого (необязательно $\boldsymbol{M}$-однородного) полинома

$$
P(z) \equiv \sum a_{k} z^{k} \not \equiv 0
$$


через $\operatorname{deg}_{\boldsymbol{M}} P$ обозначим величину $\sup |\boldsymbol{k} \boldsymbol{M}|$, где супремум берется по всем мультииндексам $\boldsymbol{k}$ с $a_{\boldsymbol{k}} \neq 0$. Если $P(\boldsymbol{z}) \equiv 0$, то $\operatorname{deg}_{\boldsymbol{M}} P:=0$. При $\boldsymbol{M}=(1, \ldots, 1)$ вместо $\operatorname{deg}_{\boldsymbol{M}} P$ будем использовать обозначение $\operatorname{deg} P$. При всех $\boldsymbol{x} \in \mathbb{R}^{n}$ и $r>0$ через $B_{\boldsymbol{M}}(\boldsymbol{x}, r)$ обозначим множество $\left\{\boldsymbol{x}+r^{\boldsymbol{M}} \boldsymbol{t}: \boldsymbol{t} \in B\right\}$, которое назовем замкнутым $\boldsymbol{M}$-шаром. Если $\boldsymbol{M}=(1, \ldots, 1)$, то будем использовать обозначение $B(\boldsymbol{x}, r)$.

Далее приводятся известные определения и результаты из теории линейных дифференциальных операторов с частньми производными. Большинство из них взято из [4] и $[5]$.

Как обычно, $\mathscr{D}^{\prime}(G)$ - пространство всех распределений в области $G ; \mathscr{E}^{\prime}(G)$ - пространство всех распределений в $G$, носитель которых компактен в этой области. Каждая локально интегрируемая в $G$ функция $f$, как известно, порождает распределение, действие которого на произвольную функцию $\varphi \in C_{0}^{\infty}(G)$ есть $\int f \varphi d x$, а каждая комплексная борелевская мера $\mu$ в $\mathbb{R}^{n}$ порождает распределение, действующее на произвольную функцию $\varphi \in C_{0}^{\infty}\left(\mathbb{R}^{n}\right)$ как $\int \varphi d \mu$, которое мы будем обозначать через $F_{\mu}$. Мы будем в дальнейшем говорить, что распределение является функцией или мерой, если оно порождается соответственно локально интегрируемой функцией или комплексной борелевской мерой в указанном выше смысле. Через $\delta$ обозначим меру Дирака - единичную меру, сосредоточенную в начале координат.

Для заданных $u \in \mathscr{D}^{\prime}\left(\mathbb{R}^{n}\right)$ и $\varphi \in C_{0}^{\infty}(G)$ свертка $u * \varphi$ есть функция класса $C^{\infty}\left(\mathbb{R}^{n}\right)$, определяемая формулой $u * \varphi(\boldsymbol{x})=u(\varphi(\boldsymbol{x}-\cdot))$, где $u(\varphi(\boldsymbol{x}-\cdot))$ обозначает действие $u$ на функцию $\varphi(\boldsymbol{x}-\boldsymbol{y})$, рассматриваемую как функция от $\boldsymbol{y}$. Если $u_{1}, u_{2} \in \mathscr{D}^{\prime}\left(\mathbb{R}^{n}\right)$ и при этом одно из этих распределений имеет компактньй носитель, то существует единственное распределение $u \in \mathscr{D}^{\prime}\left(\mathbb{R}^{n}\right)$, при котором $u_{1} *\left(u_{2} * \varphi\right)=u * \varphi$ для каждой функции $\varphi \in C_{0}^{\infty}\left(\mathbb{R}^{n}\right)$. Это распределение называется сверткой $u_{1}$ и $u_{2}$ и обозначается через $u_{1} * u_{2}$. В частности, свертка двух комплексных борелевских мер $\mu_{1}$ и $\mu_{2}$, сосредоточенных на компактах $K_{1}$ и $K_{2}$ соответственно, есть комплексная борелевская мера $\mu$, сосредоточенная на компакте $K_{1}+K_{2}:=\left\{\boldsymbol{x}+\boldsymbol{y}: \boldsymbol{x} \in K_{1}, \boldsymbol{y} \in K_{2}\right\}$ такая, что

$$
\int \varphi(\boldsymbol{x}+\boldsymbol{y}) d \mu_{1}(\boldsymbol{x}) d \mu_{2}(\boldsymbol{y})=\int \varphi(\boldsymbol{x}) d \mu(\boldsymbol{x})
$$

для каждой функции $\varphi \in C_{0}^{\infty}\left(\mathbb{R}^{n}\right)$.

Любой линейньй дифференциальньй оператор с постоянными коэффициентами $P(D)$ определяет отображение $\mathscr{D}^{\prime}(G)$ в себя: $(P(D) u)(\varphi)=u(P(-D) \varphi)$ для каждой функции $\varphi \in C_{0}^{\infty}(G)$. Если $u_{1}, u_{2} \in \mathscr{D}^{\prime}\left(\mathbb{R}^{n}\right)$ и хотя бы одно из этих распределений имеет компактньй носитель, то $P(D)\left(u_{1} * u_{2}\right)=\left(P(D) u_{1}\right) * u_{2}=u_{1} *\left(P(D) u_{2}\right)$.

Если $u, f \in \mathscr{D}^{\prime}(G), P(D) u=f$, тоговорят, что $и$ является слабым (или обобщенным) решением уравнения $P(D) g=f$ в $G$. Если $E \in \mathscr{D}^{\prime}\left(\mathbb{R}^{n}\right)$ - обобщенное решение уравнения $P(D) g=F_{\delta}$, то $E$ называется фундаментальным решением оператора $P(D)$. Для каждого линейного дифференциального оператора с постоянньпи коэффициентами $P(D)$ фундаментальное решение всегда существует. Линейньй дифференциальньй оператор с постоянными коэффициентами $P(D)$ назьвается гипоэллиптическим, если для любой области $G \subset \mathbb{R}^{n}$ и для любой функции $f \in C^{\infty}(G)$ каждое обобщенное решение уравнения $P(D) g=f$ в $G$ является функцией класса $C^{\infty}(G)$. Это равносильно тому, что оператор $P(D)$ имеет фундаментальное решение, ограничение которого на $\mathbb{R}^{n} \backslash\{\mathbf{0}\}$ есть функция класса $C^{\infty}$. Из приведенного определения легко вытекает, что 
если оператор $P(D)$ гипоэллиптичен, то оператор $P(-D)$ также гипоэллиптичен. Существуют просто формулируемые условия на полином $P=P(z), z \in \mathbb{C}^{n}$, необходимые и достаточные для того, чтобы соответствующий ему оператор $P(D)$ был гипоэллиптическим. Приведем одно из них (см. [5, с. 75]): расстояние от точки $\boldsymbol{x} \in \mathbb{R}^{n}$ до поверхности $\left\{\boldsymbol{z} \in \mathbb{C}^{n}: P(\boldsymbol{z})=0\right\}$ стремится к $\infty$ при $|\boldsymbol{x}| \rightarrow \infty$. Отсюда вытекает, в частности, что если оператор $P(D)$ гипоэллиптичен, то таковым является и каждый оператор $(P(D))^{k}, k \in \mathbb{N}_{0}$.

Преобразованием Фурье-Лапласа распределения $u \in \mathscr{E}^{\prime}\left(\mathbb{R}^{n}\right)$ назьвается функция

$$
\begin{gathered}
\widehat{u}(\boldsymbol{z}):=u\left(e^{-i(\boldsymbol{z} \cdot \boldsymbol{x})}\right), \quad \boldsymbol{z}=\left(z_{1}, \ldots, z_{n}\right) \in \mathbb{C}^{n}, \quad \boldsymbol{x}=\left(x_{1}, \ldots, x_{n}\right) \in \mathbb{R}^{n}, \\
(\boldsymbol{z} \cdot \boldsymbol{x}):=z_{1} x_{1}+\cdots+z_{n} x_{n},
\end{gathered}
$$

где распределение $u$ действует на функцию $e^{-(\boldsymbol{z} \cdot \boldsymbol{x})}$ по $\boldsymbol{x}$. В частности, для комплексной борелевской меры $\mu$ с компактным носителем в $\mathbb{R}^{n}$

$$
\widehat{F}_{\mu}(\boldsymbol{z})=\int e^{-i(\boldsymbol{z} \cdot \boldsymbol{x})} d \mu(\boldsymbol{x}) .
$$

Функция $\widehat{F}_{\mu}(\boldsymbol{z})$ назьвается преобразованием Фурье-Лапласа меры $\mu$ и обозначается через $\widehat{\mu}(\boldsymbol{z})$. Хорошо известно, что для каждого распределения $u \in \mathscr{E}^{\prime}\left(\mathbb{R}^{n}\right)$ его преобразование Фурье-Лапласа является целой функцией экспоненциального типа. Если $u \in \mathscr{E}^{\prime}\left(\mathbb{R}^{n}\right), P(D)$ - линейньй дифференциальный оператор с постоянными коэффициентами, $T(\boldsymbol{z})$ и $Q(\boldsymbol{z})$ - преобразования $\Phi$ урье-Лапласа $u$ и $P(D) u$ соответственно, то $Q(\boldsymbol{z}) \equiv P(\boldsymbol{z}) T(\boldsymbol{z})$.

Tеорема А [4, теорема 7.3.2]. Пусть $f \in \mathscr{E}^{\prime}\left(\mathbb{R}^{n}\right), P(D)$ - линейный дифференииальный оператор с постоянными коэффициентами. Уравнение $P(D) g=f$ имеет обобщенное решение $u \in \mathscr{E}^{\prime}\left(\mathbb{R}^{n}\right)$ тогда и только тогда, когда $\widehat{f}(\zeta) / P(\zeta)$ - иелая функиия. В этом случае решение однозначно определено и замыкание выпуклой оболочки носителя распределения и совпадает с замыканием выпуклой оболочки носителя распределения $f$.

Отметим, что в формулировке теоремы 7.3.2 в [4] имеется явная опечатка: вместо фразы “... $\widehat{f}(\zeta) \cdot P(\zeta)$ - целая функция" должно быть “... $\widehat{f}(\zeta) / P(\zeta)$ - целая функция".

Пусть заданы полиномы $P_{k}, k \in \mathbb{N}_{0}$. Если функция $u$ удовлетворяет в $\mathbb{R}^{n}$ равенству $P_{k}(D) u=0$ при каждом $k \in \mathbb{N}_{0}$ и

$$
u(\boldsymbol{x}) \equiv g(\boldsymbol{x}) e^{-i(\boldsymbol{z} \cdot \boldsymbol{x})}
$$

для некоторого полинома $g(\boldsymbol{x})$ и для некоторого $\boldsymbol{z} \in \mathbb{C}^{n}$, то $u$ называется әкспоненциальным решением системы $P_{k}(D) f=0, k \in \mathbb{N}_{0}$.

ЛЕмма А [4, лемма 7.3.7]. Пусть $P(D)$ - линейный дифференциальный оператор с постоянными коэффициентами. Если распределение $\nu \in \mathscr{E}^{\prime}\left(\mathbb{R}^{n}\right)$ таково, что для каждого әкспоненииального решения и уравнения $P(D) f=0$ выполнено равенство $\nu(u)=0$, то $\widehat{\nu}(\boldsymbol{z}) / P(-\boldsymbol{z})-$ челая функиия. 
ТЕорема Б [6, теорема 7.6.14]. Пусть $G$ - выпуклая область в $\mathbb{R}^{n} u P_{k}(D)$, $k=0,1, \ldots, q, q \in \mathbb{N}_{0}$, - конечный набор линейных дифференциальных операторов с постоянными коэффициентами. Тогда каждая функиия $и \in C(G)$, являющаяся в $G$ слабым решением системы $P_{k}(D) f=0, k=0,1, \ldots, q$, есть предел некоторой последовательности конечных линейных комбинаций әкспоненциальных решений этой системы, равномерно сходящейся на компактах из $G$.

2. Системы линейных дифференциальных уравнений с частными производными, определяемые обобщенным свойством среднего значения. Пусть $\boldsymbol{M}=\left(M_{1}, \ldots, M_{n}\right)$ - вектор с натуральными компонентами, $\mu$ - комплексная борелевская мера с носителем на шаре $B, G$ - область в $\mathbb{R}^{n}, n \geqslant 1$. Непрерывную в $G$ функцию $u$ будем назьвать $(\boldsymbol{M}, \mu)$-функиией (в $G)$, если

$$
\int u\left(\boldsymbol{x}+r^{\boldsymbol{M}} \boldsymbol{t}\right) d \mu(\boldsymbol{t})=0
$$

для всех $\boldsymbol{x} \in G$ и $r>0$ таких, что $B_{\boldsymbol{M}}(\boldsymbol{x}, r) \subset G$.

ТЕОрема 1. Для каждой комплексной борелевской меры н с носителем на шаре $B \subset \mathbb{R}^{n}, n \geqslant 1$, существует последовательность полиномов $\left\{P_{j}(z)\right\}_{j=0}^{\infty}, z \in \mathbb{C}^{n}$, со свойствами:

1) при каждом $j \in \mathbb{N}_{0} P_{j}(z)$ - либо $\boldsymbol{M - о д н о р о д н ы и ̆ ~ п о л и н о м , ~} \operatorname{deg}_{\boldsymbol{M}} P_{j}=j$, либо $P_{j}(z) \equiv 0$

2) для любой области $G \subset \mathbb{R}^{n}$ любая функиия $u \in C(G)$ является в $G(M, \mu)$-функиией тогда и только тогда, когда она есть слабое решение в $G$ системь $P_{j}(D) f=0, j \in \mathbb{N}_{0}$.

ДокАЗАТЕЛЬство.Пусть задана комплексная борелевская мера $\mu$ с носителемна $B$. Предположим сначала, что $и$ есть $(\boldsymbol{M}, \mu)$-функция в $\mathbb{R}^{n}$ вида (1). Фиксируя точку $\boldsymbol{x} \in \mathbb{R}^{n}$ и разлагая функцию $u$ в ряд Тейлора с центром в $\boldsymbol{x}$, а затем группируя этот ряд по $\boldsymbol{M}$-однородным полиномам, получим

$$
u(\boldsymbol{x}+\boldsymbol{y})=\sum_{j=0}^{\infty} Q_{j}(\boldsymbol{y})
$$

где

$$
Q_{j}(\boldsymbol{y})=\sum_{|\boldsymbol{k} \boldsymbol{M}|=j}(\boldsymbol{k} !)^{-1} \partial^{\boldsymbol{k}} u(\boldsymbol{x}) \boldsymbol{y}^{\boldsymbol{k}}, \quad \boldsymbol{k} !:=k_{1} ! k_{2} ! \cdots k_{n} !, \quad \boldsymbol{y}^{\boldsymbol{k}}=y_{1}^{k_{1}} \cdots y_{n}^{k_{n}}
$$

при этом ряд в правой части $(3)$ сходится к $u(\boldsymbol{x}+\boldsymbol{y})$ равномерно на каждом компакте из $\mathbb{R}^{n}$. Зафиксируем произвольным образом $r>0$ и положим в (3) $\boldsymbol{y}=r^{\boldsymbol{M}} \boldsymbol{t}$. Интегрируя почленно обе части полученного равенства по мере $\mu$, что законно в силу равномерной сходимости ряда из (3), получаем

$$
\int u\left(\boldsymbol{x}+r^{\boldsymbol{M}} \boldsymbol{t}\right) d \mu(\boldsymbol{t})=\sum_{j=0}^{\infty} \int Q_{j}\left(r^{\boldsymbol{M}} \boldsymbol{t}\right) d \mu(\boldsymbol{t})=\sum_{j=0}^{\infty} r^{j}\left(P_{j}(D) u\right)(\boldsymbol{x})
$$


где

$$
P_{j}(\boldsymbol{z}) \equiv \sum_{|\boldsymbol{k} M|=j}(\boldsymbol{k} !)^{-1}(i \boldsymbol{z})^{\boldsymbol{k}} \int \boldsymbol{t}^{\boldsymbol{k}} d \mu(\boldsymbol{t}), \quad j \in \mathbb{N}_{0}, \quad z \in \mathbb{C}^{n} .
$$

Поскольку и есть $(\boldsymbol{M}, \mu)$-функция в $\mathbb{R}^{n}$, в силу произвола в выборе $\boldsymbol{x}$ и $r$ из (4) вытекает, что $P_{j}(D) u(\boldsymbol{x}) \equiv 0, j \in \mathbb{N}_{0}, \boldsymbol{x} \in \mathbb{R}^{n}$. Покажем, что последовательность полиномов $\left\{P_{j}(\boldsymbol{z})\right\}_{j=0}^{\infty}$, определенных формулой $(5)$, удовлетворяет условиям теоремы.

Пусть $G$ - произвольная область в $\mathbb{R}^{n}$. Зафиксируем каким-либо образом функцию $\varphi \in C_{0}^{\infty}(G)$ и $\boldsymbol{M}$-шар $B_{\boldsymbol{M}}(\boldsymbol{x}, r) \subset G, r>0$. По формуле Тейлора с остаточным членом в интегральной форме при каждом $l \in \mathbb{N}_{0}$ и при всех $\boldsymbol{y} \in B_{\boldsymbol{M}}(0, r)$ имеем равенство

$$
\varphi(\boldsymbol{x}+\boldsymbol{y})=\sum_{|\boldsymbol{k}|<l}(\boldsymbol{k} !)^{-1} \partial^{\boldsymbol{k}} \varphi(\boldsymbol{x}) \boldsymbol{y}^{\boldsymbol{k}}+l \int_{0}^{1}(1-s)^{l-1}\left(\sum_{|\boldsymbol{k}|=l}(\boldsymbol{k} !)^{-1} \partial^{\boldsymbol{k}} \varphi(\boldsymbol{x}+s \boldsymbol{y}) \boldsymbol{y}^{\boldsymbol{k}}\right) d s
$$

Полагая $\boldsymbol{y}=-r^{\boldsymbol{M}} \boldsymbol{t}, \boldsymbol{t} \in \boldsymbol{B}$, и перегруппировывая слагаемые, получаем

$$
\varphi\left(\boldsymbol{x}-r^{\boldsymbol{M}} \boldsymbol{t}\right)=\sum_{j=0}^{p} r^{j}\left(\sum_{|\boldsymbol{k} M|=j}(-1)^{|\boldsymbol{k}|}(\boldsymbol{k} !)^{-1} \partial^{\boldsymbol{k}} \varphi(\boldsymbol{x}) \boldsymbol{t}^{\boldsymbol{k}}\right)+R_{p}(r, \boldsymbol{x}, \boldsymbol{t}),
$$

где $p=p(l) \in \mathbb{N}_{0}$ - наибольшее число, при котором для любого мультииндекса $\boldsymbol{k}$ из $|\boldsymbol{k} \boldsymbol{M}| \leqslant p$ вытекает, что $|\boldsymbol{k}|<l ; R_{p}(r, \boldsymbol{x}, \boldsymbol{t})=o\left(r^{p}\right)$ при $r \rightarrow 0$ равномерно по $\boldsymbol{x} \in \operatorname{supp} \varphi$ и $\boldsymbol{t} \in B$. Ясно, что на самом деле (6) выполняется при каждом $p \in \mathbb{N}_{0}$. Интегрируя обе части (6) по мере $\mu$, получаем

$$
\int \varphi\left(\boldsymbol{x}-r^{\boldsymbol{M}} \boldsymbol{t}\right) d \mu(\boldsymbol{t})=\sum_{j=0}^{p} r^{j}\left(\sum_{|\boldsymbol{k} \boldsymbol{M}|=j}(-1)^{|\boldsymbol{k}|}(\boldsymbol{k} !)^{-1} \partial^{\boldsymbol{k}} \varphi(\boldsymbol{x}) \int \boldsymbol{t}^{\boldsymbol{k}} d \mu(\boldsymbol{t})\right)+W_{p}(r, \boldsymbol{x})
$$

или

$$
\int \varphi\left(\boldsymbol{x}-r^{\boldsymbol{M}} \boldsymbol{t}\right) d \mu(\boldsymbol{t})=\sum_{j=0}^{p} r^{j}\left(P_{j}(-D) \varphi\right)(\boldsymbol{x})+W_{p}(r, \boldsymbol{x})
$$

где

$$
P_{j}(-D)=\sum_{|\boldsymbol{k} M|=j}(-1)^{|\boldsymbol{k}|}(\boldsymbol{k} !)^{-1}(i D)^{\boldsymbol{k}} \int \boldsymbol{t}^{\boldsymbol{k}} d \mu(\boldsymbol{t}),
$$

$W_{p}(r, \boldsymbol{x})=o\left(r^{p}\right)$ при $r \rightarrow 0$ равномерно по $\boldsymbol{x} \in \operatorname{supp} \varphi$.

Пусть теперь непрерывная в $G$ функция $u$ является в этой области $(\boldsymbol{M}, \mu)$-функцией, т.е. для всех $\boldsymbol{x} \in G$ и $r>0$ таких, что $B_{\boldsymbol{M}}(\boldsymbol{x}, r) \subset G$, выполнено равенство (2). Умножая это равенство на $\varphi(\boldsymbol{x})$ и интегрируя по мере Лебега в $G$, получаем (при достаточно малых $r$ )

$$
\begin{aligned}
0 & =\int\left(\int u\left(\boldsymbol{x}+r^{\boldsymbol{M}} \boldsymbol{t}\right) d \mu(\boldsymbol{t})\right) \varphi(\boldsymbol{x}) d \boldsymbol{x}=\int u(\boldsymbol{x})\left(\int \varphi\left(\boldsymbol{x}-r^{\boldsymbol{M}} \boldsymbol{t}\right) d \mu(\boldsymbol{t})\right) d \boldsymbol{x} \\
& =\int\left(u(\boldsymbol{x})\left(\sum_{j=0}^{p} r^{j}\left(P_{j}(-D) \varphi\right)(\boldsymbol{x})\right)+W_{p}(r, \boldsymbol{x})\right) d \boldsymbol{x} \\
& =\sum_{j=0}^{p} r^{j}\left(\int u(\boldsymbol{x})\left(P_{j}(-D) \varphi\right)(\boldsymbol{x}) d \boldsymbol{x}\right)+o\left(r^{p}\right), \quad r \rightarrow 0 .
\end{aligned}
$$


(Мы последовательно сделали замены $\boldsymbol{y}=\boldsymbol{x}+r^{\boldsymbol{M}} \boldsymbol{t}$ и $\boldsymbol{x}=\boldsymbol{y}$, воспользовались теоремой Фубини и равенством (7), а также тем, что $W_{p}(r, \boldsymbol{x})=o\left(r^{p}\right)$ при $r \rightarrow 0$ равномерно по $\boldsymbol{x} \in \operatorname{supp} \varphi$.) Предположим, что хотя бы один из полиномов (5) не равен тождественно нулю. Тогда, полагая $p$ в (8) наименьшим целым неотрицательным числом, при котором $P_{p}(\boldsymbol{z}) \not \equiv 0$, затем, поделив последнюю строчку (8) на $r^{p}$ и устремив $r$ к 0, мы получим, что $\int u(\boldsymbol{x})\left(P_{p}(-D) \varphi\right)(\boldsymbol{x}) d \boldsymbol{x}=0$. Отсюда по индукции следует, что $\int u(\boldsymbol{x})\left(P_{j}(-D) \varphi\right)(\boldsymbol{x}) d \boldsymbol{x}=0$ при всех $j \in \mathbb{N}_{0}$. Если все полиномы в (5) равны тождественно нулю, то последнее утверждение очевидно. В наших рассуждениях функция $\varphi \in C_{0}^{\infty}(G)$ может быть фиксирована произвольным образом, значит $u$ является в $G$ слабым решением системы

$$
P_{j}(D) f=0, \quad j \in \mathbb{N}_{0} .
$$

Обратно, пусть непрерывная в $G$ функция $u$ является в этой области слабым решением системы (9). Мы хотим показать, что $u$ есть $(\boldsymbol{M}, \mu)$-функция в $G$. Полиномы $P_{j}(\boldsymbol{z}), j \in \mathbb{N}_{0}$, порождают идеал $\mathfrak{U}$ в кольце всех многочленов от $n$ переменных над полем $\mathbb{C}$. По теореме Гильберта о базисе (см. [7, с. 421-423]) идеал $\mathfrak{U}$ конечно порожден, следовательно, существует $q \in \mathbb{N}_{0}$ такое, что каждьй полином $L \in \mathfrak{U}$ представим в виде $L=\sum_{j=0}^{q} L_{j} P_{j}$ с некоторьми полиномами $L_{j}, j=0,1, \ldots, q$. Это означает, что система (9) эквивалентна системе дифференциальных уравнений $P_{k}(D) f=0$, $k=0,1, \ldots, q$. Отсюда и из теоремы Б следует, что для доказательства того, что $u$ есть $(\boldsymbol{M}, \mu)$-функция в $G$, можно, не теряя общности, считать, что $G=\mathbb{R}^{n}$ и $u$ - экспоненциальное решение системы $P_{k}(D) f=0, k \in \mathbb{N}_{0}$.

Возьмем произвольно $\boldsymbol{M}$-шар $B_{\boldsymbol{M}}(\boldsymbol{x}, r) \subset \mathbb{R}^{n}, r>0$. Тогда функция $u(\boldsymbol{x}+\boldsymbol{y})$, $\boldsymbol{y} \in B_{\boldsymbol{M}}(\mathbf{0}, r)$, разлагается в ряд $(3)$, сходящийся к $u(\boldsymbol{x}+\boldsymbol{y})$ равномерно на $B_{\boldsymbol{M}}(\mathbf{0}, r)$, и замена $\boldsymbol{y}=r^{\boldsymbol{M}} \boldsymbol{t}$ с почленньм интегрированием полученного ряда (см. формулу (4)) дает равенство (2). Таким образом, $u$ есть $(\boldsymbol{M}, \mu)$-функция в $\mathbb{R}^{n}$. Теорема доказана.

3. Теорема о среднем для слабых решений $M$-однородных линейных дифференциальных уравнений с частными производными. Пусть $\boldsymbol{M}=\left(M_{1}, \ldots\right.$, $M_{n}$ ) - тот же вектор, что и вьше, $\mu$ - комплексная борелевская мера с носителем на единичном шаре $B, P(D)$ - линейный дифференциальньй оператор с постоянными коэффициентами в $\mathbb{R}^{n}, n \geqslant 1$. Будем говорить, что мера $\mu$ и (целочисленньй) вектор $\boldsymbol{M}$ характеризуют решения уравнения $P(D) f=0$, если для любой области $G \subset \mathbb{R}^{n}$ и любой функции $u \in C(G)$ эквивалентны следуюшие условия:

а) $и$ есть $(\boldsymbol{M}, \mu)$-функция в $G$;

б) $u$ - слабое решение уравнения $P(D) f=0$ в $G$.

В принятых обозначениях имеет место следующая

Теорема 2. Для того чтобы мера $\mu$ и вектор $\boldsymbol{M}$ характеризовали решения уравнения $P(D) f=0$, необходимо и достаточно, чтобы оператор $P(D)$ был $M$-однородным, а $F_{\mu}=P(-D) T$, где $T$ - некоторое распределение с носителем на $B, \widehat{T}(\mathbf{0}) \neq 0$.

Доказательству этой теоремы предпошлем лемму, которая фактически установлена в работе [1].

Лемма 1. Пусть даны полиномы $P(\boldsymbol{z}) \not \equiv 0$ u $P_{j}(\boldsymbol{z}), j \in \mathbb{N}_{0}, \boldsymbol{z} \in \mathbb{C}^{n}$, такие, что при каждом $j \in \mathbb{N}_{0}$ либо $P_{j}(z)-M$-однородный полином, $\operatorname{deg}_{M} P_{j}=j$, либо 
$P_{j}(\boldsymbol{z}) \equiv 0 ; n р и$ этом $P_{k}(\boldsymbol{z}) \not \equiv 0$ хотя бы для одного номера $k \in \mathbb{N}_{0}$. Система дифференциальных уравнений (9) эквивалентна уравнению $P(D) f=0$ тогда и только тогда, когда полином $P(\boldsymbol{z})$ делит каждый из полиномов $P_{j}(\boldsymbol{z}), j \in \mathbb{N}_{0}, u$ для какого-то номера $k \in \mathbb{N}_{0}$ полином $P_{k}(\boldsymbol{z})$ с точностью до ненулевого постоянного множителя совпадает с полиномом $P(z)$.

ДокаЗАТЕЛЬСтво. Фиксируем $j \in \mathbb{N}_{0}$, чтобы $P_{j}(\boldsymbol{z}) \not \equiv 0$. Пусть $K(\boldsymbol{z})$ - (каким-либо образом фиксированньй) наибольший общий делитель полиномов $P_{j}(\boldsymbol{z})$ и $P(\boldsymbol{z})$ такой, что $P_{j}(\boldsymbol{z}) \equiv K(\boldsymbol{z}) Q_{j}(\boldsymbol{z}), P(\boldsymbol{z}) \equiv K(\boldsymbol{z}) L(\boldsymbol{z})$, где $Q_{j}(\boldsymbol{z})$ и $P(\boldsymbol{z})$ - некоторые полиномы. При каждом $\boldsymbol{z} \in \mathbb{C}^{n}$ уравнение $K(D) f=e^{i(\boldsymbol{z} \cdot \boldsymbol{x})}$ имеет в $\mathbb{R}^{n}$ решение $g \in C^{\infty}\left(\mathbb{R}^{n}\right)$ (см. [5, следствие 10.6.8]). Значит, если $L(\boldsymbol{z})=0$, то

$$
P(D) g=L(D) K(D) g=L(D) e^{i(\boldsymbol{z} \cdot \boldsymbol{x})}=L(\boldsymbol{z}) e^{i(\boldsymbol{z} \cdot \boldsymbol{x})}=0,
$$

откуда

$$
P_{j}(D) g=Q_{j}(D) K(D) g=Q_{j}(\boldsymbol{z}) e^{i(\boldsymbol{z} \cdot \boldsymbol{x})}=0 .
$$

Таким образом, $Q_{j}(\boldsymbol{z})=0$ во всех точках $\boldsymbol{z} \in \mathbb{C}^{n}$, в которых $L(\boldsymbol{z})=0$. По теореме Гильберта о нулях (см. [7, с. 468]) существует $m \in \mathbb{N}_{0}$ такое, что полином $Q_{j}^{m}$ делится на полином $L$, но $Q_{j}$ и $L$ не имеют общих делителей, отличных от постоянных; следовательно, полином $L$ - ненулевая постоянная (если бы $L$ содержал в качестве делителя неприводимьй полином ненулевой степени, то последний, очевидно, был бы делителем $Q_{j}$, что противоречит взаимной простоте $L$ и $Q_{j}$ ). Значит, $P_{j}$ делится на $P$. Легко видеть, что любой $\boldsymbol{M}$-однородньй полином, тождественно не равньй нулю, в качестве делителей имеет только $\boldsymbol{M}$-однородные полиномы, откуда вытекает, что полином $P$ $\boldsymbol{M}$-однородный. Выберем из полиномов $P_{j}, j \in \mathbb{N}_{0}$, тождественно не равньй нулю полином с наименьшим номером $j$. Пусть для определенности это номер $k$. Поскольку $P_{k}$ делится на $P$, частное от этого деления $S$ есть некоторый $\boldsymbol{M}$-однородньй полином. Если $\operatorname{deg}_{M} S>0$, то всякое бесконечно дифференцируемое решение уравнения $P(D) f=1$ в $\mathbb{R}^{n}$ должно быть решением системы (9), что невозможно. Значит, $\operatorname{deg}_{M} S=0$ и полином $S$ - ненулевая постоянная. Лемма доказана.

ДоКАЗАтеЛЬСтво теоРемЫ 2. Необходимость. Рассмотрим преобразование Фурье-Лапласа меры $\mu$ :

$$
\widehat{\mu}(\boldsymbol{z})=\int e^{-i(\boldsymbol{z} \cdot \boldsymbol{t})} d \mu(\boldsymbol{t}) .
$$

Поскольку $\widehat{\mu}(\boldsymbol{z})$ - целая функция, ряд Тейлора этой функции с центром в точке $\boldsymbol{z}=\mathbf{0}$ сходится абсолютно и равномерно на каждом компакте из $\mathbb{C}^{n}$. Следовательно, сгруппировав этот ряд по $\boldsymbol{M}$-однородным полиномам в порядке возрастания $\boldsymbol{M}$-степени, мы получим ряд, равномерно сходящийся к $\widehat{\mu}(\boldsymbol{z})$ на компактных подмножествах из $\mathbb{C}^{n}$ :

$$
\begin{aligned}
\widehat{\mu}(\boldsymbol{z}) & =\int e^{-i(\boldsymbol{z} \cdot \boldsymbol{t})} d \mu(\boldsymbol{t})=\sum_{k=0}^{\infty}(k !)^{-1} \int(-i(\boldsymbol{z} \cdot \boldsymbol{t}))^{k} d \mu(\boldsymbol{t}) \\
& =\sum_{k=0}^{\infty}(-i)^{k}(k !)^{-1}\left(\sum_{|\boldsymbol{l}|=k} C_{k}^{\boldsymbol{l}} \boldsymbol{z}^{\boldsymbol{l}} \int \boldsymbol{t}^{\boldsymbol{l}} d \mu(\boldsymbol{t})\right)=\sum_{\boldsymbol{l}}\left((\boldsymbol{l} !)^{-1}(-i \boldsymbol{z})^{\boldsymbol{l}} \int \boldsymbol{t}^{\boldsymbol{l}} d \mu(\boldsymbol{t})\right) \\
& =\sum_{j=0}^{\infty}\left(\sum_{|\boldsymbol{l} \boldsymbol{M}|=j}(\boldsymbol{l} !)^{-1}(-i \boldsymbol{z})^{\boldsymbol{l}} \int \boldsymbol{t}^{\boldsymbol{l}} d \mu(\boldsymbol{t})\right)=\sum_{j=0}^{\infty} P_{j}(-\boldsymbol{z}),
\end{aligned}
$$


где

$$
\begin{gathered}
\boldsymbol{l}=\left(l_{1}, \ldots, l_{n}\right) \in \mathbb{N}_{0}^{n}, \quad C_{k}^{\boldsymbol{l}}:=\frac{k !}{l_{1} ! \cdots l_{n} !}, \quad l !:=l_{1} ! \cdots l_{n} !, \\
P_{j}(\boldsymbol{z}) \equiv \sum_{|\boldsymbol{l} \boldsymbol{M}|=j}(\boldsymbol{l} !)^{-1}(i \boldsymbol{z})^{\boldsymbol{l}} \int \boldsymbol{t}^{\boldsymbol{l}} d \mu(\boldsymbol{t}), \quad j \in \mathbb{N}_{0} .
\end{gathered}
$$

Если мера $\mu$ и вектор $\boldsymbol{M}$ характеризуют решения уравнения $P(D) f=0$, то, повторяя рассуждения, проведенные при доказательстве теоремы 1 , мы получаем, что это уравнение должно быть эквивалентно системе дифференциальных уравнений $P_{j}(D) f=0$, $j \in \mathbb{N}_{0}$. Предположим, что $P(\boldsymbol{z}) \not \equiv 0$ (в противном случае утверждение теоремы тривиально). Ясно, что $P_{j}(\boldsymbol{z}) \not \equiv 0$ хотя бы для одного номера $j \in \mathbb{N}_{0}$. Поэтому из леммы 1 следует $\boldsymbol{M}$-однородность оператора $P(D)$.

Из того, что мера $\mu$ и вектор $\boldsymbol{M}$ характеризуют решения уравнения $P(D) f=0$, и из леммы А вытекает, что $S(\boldsymbol{z}):=\widehat{\mu}(\boldsymbol{z}) / P(-\boldsymbol{z})$ - целая функция. По теореме А отсюда получаем, что $F_{\mu}=P(-D) T$ для некоторого распределения $T$ с носителем на $B$. Выполняя для обеих частей последнего равенства преобразование Фурье-Лапласа, мы видим, что $\widehat{\mu}(\boldsymbol{z}) \equiv P(-\boldsymbol{z}) \widehat{T}(\boldsymbol{z})$. Следовательно, $\widehat{T}(\boldsymbol{z}) \equiv S(\boldsymbol{z})$. Далее, из леммы 1 и единственности представления целой функции равномерно сходящимся на компактах из $\mathbb{C}^{n}$ рядом по $\boldsymbol{M}$-однородным полиномам с центром в точке $\boldsymbol{z}=\mathbf{0}$ получаем, что $\widehat{T}(\mathbf{0}) \neq 0$.

Достаточность. Пусть $P(D)-M$-однородньй оператор, а мера $\mu$ такова, что $F_{\mu}=$ $P(-D) T$, где $T$ - распределение с носителем на $B$, при этом необязательно, чтобы $\widehat{T}(\mathbf{0}) \neq 0$. Пусть $u$ - экспоненциальное решение уравнения $P(D) f=0\left(\right.$ в $\left.\mathbb{R}^{n}\right)$. Если $\widehat{T}(\boldsymbol{z})=\sum_{j=0}^{\infty} Q_{j}(\boldsymbol{- z})$ - разложение целой функции $\widehat{T}$ в сгруппированный по $\boldsymbol{M}$-однородным полиномам ряд Тейлора с центром в точке $\boldsymbol{z}=\mathbf{0}\left(\operatorname{deg}_{j} Q=j\right)$, то, сравнивая равенства

$\widehat{\mu}(\boldsymbol{z}) \equiv P(-\boldsymbol{z}) \widehat{T}(\boldsymbol{z}), \quad \widehat{\mu}(\boldsymbol{z}) \equiv \sum_{j=0}^{\infty} P_{j}(-\boldsymbol{z}), \quad \int u\left(\boldsymbol{x}+r^{\boldsymbol{M}} \boldsymbol{t}\right) d \mu(\boldsymbol{t})=\sum_{j=0}^{\infty} r^{j}\left(P_{j}(D) u\right)(\boldsymbol{x})$ (см. формулу (4)), а такжеполагая $m=\operatorname{deg}_{\boldsymbol{M}} P$, мы получаем, что $P_{j}(\boldsymbol{z}) \equiv 0$ при $j<m$ и $P_{j+m}(\boldsymbol{z}) \equiv P(\boldsymbol{z}) Q_{j}(\boldsymbol{z}), j \in \mathbb{N}_{0}$. Следовательно,

$$
\int u\left(\boldsymbol{x}+r^{\boldsymbol{M}} \boldsymbol{t}\right) d \mu(\boldsymbol{t})=\sum_{j=0}^{\infty} r^{j+m}\left(Q_{j}(D) P(D) u\right)(\boldsymbol{x})=0,
$$

что с учетом теоремы Б полностью доказьвает теорему 2.

ЗАмечаниЕ 1 . Теоремы 1 и 2 можно обобщить следующим образом. Пусть $\boldsymbol{M}=$ $\left(M_{1}, \ldots, M_{n}\right)$ - тот же вектор, что и вьше, $\mu$ - комплексная борелевская мера (в $\left.\mathbb{R}^{n}\right)$ с компактным носителем, $G$ - область в $\mathbb{R}^{n}$. Будем назьвать $(\boldsymbol{M}, \mu)$-функиией $($ в $G)$ всякую функцию $u \in C(G)$, для которой (2) вьполнено при всех $\boldsymbol{x} \in G$ и $r>0$ таких, что $\left\{\boldsymbol{x}+r^{\boldsymbol{M}} \boldsymbol{t}: \boldsymbol{t} \in \operatorname{supp} \mu\right\} \subset G$. Пусть $P(D)$ - линейньй дифференциальньй оператор с постоянными коэффициентами. Будем говорить, что мера $\mu$ и вектор $\boldsymbol{M}$ характеризуют решения уравнения $P(D) f=0$, если для любой области $G \subset \mathbb{R}^{n}$ и любой функции $u \in C(G)$ эквивалентны условия а), б), сформулированные в начале п. 3 , но $(\boldsymbol{M}, \mu)$-функция в $G$ понимается в указанном только что смысле.

При таком определении теорема 1 остается справедливой, а если supp $\mu$ - вьпуклое множество, то верна и теорема 2 , в ее формулировке нужно лишь заменить шар $B$ на supp $\mu$ (требование вьпуклости возникает в результате применения теоремы A). 
СЛЕДСТВИЕ 1. Для каждого $\boldsymbol{M}$-однородного линейного дифференииального оператора с постоянными коэффичиентами $P(D)$ существует комплексная борелевская мера $\mu$ с носителем на $B$, абсолютно непрерывная относительно меры Лебега, которая вместе с вектором $M$ характеризует решения уравнения $P(D) f=0$.

ДокАЗАтЕльство. Возьмем функцию $f \in C_{0}^{\infty}(B)$ такую, что $\widehat{f}(\mathbf{0})=\int f(\boldsymbol{x}) d \boldsymbol{x} \neq 0$. Тогда мера $\mu: d \mu(\boldsymbol{x})=(P(-D) f)(\boldsymbol{x}) d \boldsymbol{x}$ является требуемой.

СледСТВИЕ 2. Пусть $P_{1}(D)$ и $P_{2}(D)$ - $M$-однородные линейные дифференциальные операторы с постоянными коэффициентами и комплексные борелевские меры $\mu_{1}$ и $\mu_{2}$ с носителями на $B$ характеризуют вместе с вектором $\boldsymbol{M}$ решения уравнений $P_{1}(D) f=0$ и $P_{2}(D) f=0$ соответственно. Тогда для любой обласmи $G \subset \mathbb{R}^{n}$ любая функиия $u \in C(G)$ является в $G$ слабым решением уравнения $P_{1}(D) P_{2}(D) f=0$ в том и только том случае, когда

$$
\int u\left(\boldsymbol{x}+r^{\boldsymbol{M}} \boldsymbol{t}+r^{\boldsymbol{M}} \boldsymbol{s}\right) d \mu_{1}(\boldsymbol{t}) d \mu_{2}(\boldsymbol{s})=0
$$

при всех $\boldsymbol{x} \in G$ и $r>0$ таких, что $\left\{\boldsymbol{x}+r^{\boldsymbol{M}} \boldsymbol{t}: \boldsymbol{t} \in B(\mathbf{0}, 2)\right\} \subset G$.

ДокАЗАТЕЛЬСТво. Из теоремы 2 вытекает, что $F_{\mu_{j}}=P_{j}(-D) T_{j}$, где $T_{j}$-некоторое распределение с носителем на $B, \widehat{T}_{j}(\mathbf{0}) \neq 0, j=1,2$. Пусть $\mu=\mu_{1} * \mu_{2}$ и $T=T_{1} * T_{2}$. Из свойств свертки (см. п. 1) следует, что $\mu$-комплексная борелевская мера с носителем на шаре $B(\mathbf{0}, 2), T$ - распределение с носителем на $B(\mathbf{0}, 2), \widehat{T}(\mathbf{0})=\widehat{T}_{1}(\mathbf{0}) \widehat{T}_{2}(\mathbf{0}) \neq 0$,

$$
\begin{gathered}
F_{\mu}=\left(P_{1}(-D) T_{1}\right) *\left(P_{2}(-D) T_{2}\right)=P_{1}(-D) P_{2}(-D)\left(T_{1} * T_{2}\right)=P_{1}(-D) P_{2}(-D) T \\
\int u\left(\boldsymbol{x}+r^{\boldsymbol{M}} \boldsymbol{t}+r^{\boldsymbol{M}} \boldsymbol{s}\right) d \mu_{1}(\boldsymbol{t}) d \mu_{2}(\boldsymbol{s})=\int u\left(\boldsymbol{x}+r^{\boldsymbol{M}} \boldsymbol{t}\right) d \mu(\boldsymbol{t})
\end{gathered}
$$

Последние равенства с учетом замечания 1 дают необходимое утверждение.

СлЕДСТВИЕ 3. Пусть $P(D)-M$-однородный линейный дифференциальный оператор с постоянными коэффициентами, $\mu$ - комплексная борелевская мера $c$ носителем на $B$, характеризующая вместе с вектором $\boldsymbol{M}$ решения уравнения $P(D) f=0, k \in \mathbb{N}$. Для любой области $G \subset \mathbb{R}^{n}$ любая функиия $и \in C(G)$ является в $G$ слабым решением уравнения $(P(D))^{k} f=0$ тогда и только тогда, когда

$$
\begin{gathered}
\int u\left(\boldsymbol{x}+r^{\boldsymbol{M}} \boldsymbol{t}_{1}+r^{\boldsymbol{M}} \boldsymbol{t}_{2}+\cdots+r^{\boldsymbol{M}} \boldsymbol{t}_{k}\right) d \mu\left(\boldsymbol{t}_{1}\right) d \mu\left(\boldsymbol{t}_{2}\right) \cdots d \mu\left(\boldsymbol{t}_{k}\right)=0, \\
\boldsymbol{t}_{j}=\left(t_{j 1}, \ldots, t_{j n}\right) \in \mathbb{R}^{n}, \quad j=1, \ldots, k,
\end{gathered}
$$

для всех $\boldsymbol{x} \in G$ u $r>0$ maких, ито $\left\{\boldsymbol{x}+r^{\boldsymbol{M}} \boldsymbol{t}: \boldsymbol{t} \in B(\mathbf{0}, k R)\right\} \subset G$.

Это следствие вытекает из следствия 2 по индукции.

Дадим определение, необходимое для формулировки следующего следствия из теоремы 2. Пусть $\rho$ - функция из $L_{1}\left(\mathbb{R}^{n}\right)$, равная 0 вне шара $B, \int \rho(\boldsymbol{t}) d \boldsymbol{t}=1, P(D)$ - линейньй дифференциальный оператор с постоянными коэффициентами. Будем говорить, 
что $(M, \rho)$-регуляризачия сохраняет решения уравнения $P(D) f=0$, если для любой области $G \subset \mathbb{R}^{n}$ каждая функция $u \in C(G)$, являющаяся в $G$ слабым решением уравнения $P(D) f=0$, удовлетворяет равенству

$$
\int u\left(\boldsymbol{x}+r^{\boldsymbol{M}} \boldsymbol{t}\right) \rho(\boldsymbol{t}) d \boldsymbol{t}=u(\boldsymbol{x})
$$

при всех $\boldsymbol{x} \in G$ и $r>0$ таких, что $B_{\boldsymbol{M}}(\boldsymbol{x}, r) \subset G$. Если в дополнение к этому для любой области $G \subset \mathbb{R}^{n}$ и для любой функции $u \in C(G)$ из справедливости равенства (10) при всех указанных вьше $\boldsymbol{x}$ и $r$ следует, что $u$-слабое решение уравнения $P(D) f=0$ в области $G$, то мы будем говорить, что $(\boldsymbol{M}, \rho)$-регуляризачия характеризует решения уравнения $P(D) f=0$.

СлЕДСТВИЕ 4. Пусть $P(D)$ - гипоэллиптический $\boldsymbol{M - о д н о р о д н ы и ̆ ~ л и н е и ̆ н ы и ̆ ~ д и ф - ~}$ ференциальный оператор с постоянными коәффициентами, $\operatorname{deg}_{M} P>0$. Тогда существует функиия $\rho \in C_{0}^{\infty}(B)$ такая, что $(\boldsymbol{M}, \rho)$-регуляризация характеризует решения уравнения $P(D) f=0$.

ДокАЗАТЕЛЬСТво. Пусть $E$ - фундаментальное решение гипоэллиптического опеpaтора $P(-D)$ (напомним, что $E$ - бесконечно дифференцируемая функция в $\mathbb{R}^{n} \backslash\{\mathbf{0}\}$ ). Возьмем функцию $g \in C_{0}^{\infty}(B)$ такую, что $g(\boldsymbol{x})=1$ при всех $\boldsymbol{x} \in B(\mathbf{0}, 1 / 2)$ и $\widehat{g E}(\mathbf{0}) \neq 0$. Тогда

$$
P(-D)(-g E)=P(-D)((1-g) E)-P(-D) E=P(-D)((1-g) E)-F_{\delta}
$$

в $\mathscr{D}^{\prime}\left(\mathbb{R}^{n}\right)$, причем распределение $P(-D)((1-g) E)$ - бесконечно дифференцируемая функция с носителем в кольце $\{1 / 2 \leqslant|x| \leqslant 1\}$. Из теоремы 2 вытекает, что функция $\rho(\boldsymbol{x}):=P(-D)((1-g) E)(\boldsymbol{x})$ обладает требуемьми свойствами.

Из известных критериев гипоэллиптичности (см., например, [5, с. 75]) вытекает, что $\boldsymbol{M}$-однородный линейньй дифференциальньй оператор с постоянными коэффициентами $P(D)$ является гипоэллиптическим тогда и только тогда, когда $P(\boldsymbol{x}) \neq 0$ для любого $\boldsymbol{x} \in \mathbb{R}^{n} \backslash\{\boldsymbol{0}\}$; откуда следует, что $\boldsymbol{M}$-однородные гипоэллиптические операторы являются частным случаем так назьваемых квазиэллиптических операторов, введенных Л.Р. Волевичем [8] и более известных как полуэллиптические операторы. Отметим (без доказательства), что из теоремы 2 можно вьвести теорему о среднем для решений уравнения теплопроводности, установленную У. Фалксом в работе [2].

СлЕДСТВИЕ 5. Пусть $P(D)-M$-однородный гипоэллиптический линейный дифференциальный оператор с постоянными коэффициентами в $\mathbb{R}^{n}, n \geqslant 2, \operatorname{deg}_{M} P=$ $m>0, E-$ фундаментальное решение оператора $P(D)$. Тогда

1) если $|\boldsymbol{M}|<m$, mo $E \in L_{\infty}\left(\mathbb{R}^{n} ;\right.$ loc $)$;

2) если $|\boldsymbol{M}|=m$, то $E \in L_{p}\left(\mathbb{R}^{n}\right.$; loc) при каждом $p<\infty$;

3) если $|\boldsymbol{M}|>m$, то $E \in L_{p}\left(\mathbb{R}^{n} ;\right.$ loc $)$ при каждом $p<|\boldsymbol{M}|(|\boldsymbol{M}|-m)^{-1}$.

ДокАЗАТЕЛЬСТво. Существует постоянная $K_{1}=K_{1}(P)$ такая, что для любой функции $\Phi \in C_{0}^{\infty}\left(\mathbb{R}^{n}\right)$ с носителем, принадлежащим некоторому $\boldsymbol{M}$-шару $B_{\boldsymbol{M}}(\boldsymbol{x}, r), r>0$, выполняется неравенство

$$
\|\Phi\|_{2} \leqslant K_{1} r^{m}\|P(D) \Phi\|_{2} \text {. }
$$


Если $\boldsymbol{x}=\mathbf{0}$ и $r=1$, то сформулированное утверждение есть частньй случай одной леммы Л. Хёрмандера (см. [9, с. 510]), а случай произвольных $\boldsymbol{x}$ и $r$ сводится к $\boldsymbol{x}=\boldsymbol{0}$ и $r=1$ с помощью функции $\Phi_{\boldsymbol{x}, r}(\boldsymbol{y}):=\Phi\left(\boldsymbol{x}+r^{\boldsymbol{M}} \boldsymbol{y}\right)$. Пусть функция $\rho \in C_{0}^{\infty}(B), \int \rho(\boldsymbol{t}) d \boldsymbol{t}=1$, такова, что $(\boldsymbol{M}, \rho)$-регуляризация сохраняет решения уравнения $P(D) f=0$. Положим $\rho_{s}(\boldsymbol{t}):=s^{-|\boldsymbol{M}|} \rho\left(-s^{\boldsymbol{M}} \boldsymbol{t}\right)$ и $\lambda_{s}(\boldsymbol{t}):=\rho_{s}(\boldsymbol{t})-\rho_{s / 2}(\boldsymbol{t}), s>0, \boldsymbol{t} \in \mathbb{R}^{n}$, и рассмотрим семейство функций $g_{s}(\boldsymbol{y}):=E * \rho_{s}(\boldsymbol{y}), \boldsymbol{y} \in \mathbb{R}^{n}$. Несложно проверить, что $g_{s}-g_{s / 2} \in$ $C_{0}^{\infty}\left(B_{M}(\mathbf{0}, s)\right)$. Из неравенства Гёльдера и (11) получаем, что

$$
\begin{aligned}
\left\|g_{s}-g_{s / 2}\right\|_{1} & \leqslant K_{2} s^{|\boldsymbol{M}| / 2}\left\|g_{s}-g_{s / 2}\right\|_{2} \leqslant K_{1} K_{2} s^{|\boldsymbol{M}| / 2} s^{m}\left\|P(D)\left(g_{s}-g_{s / 2}\right)\right\|_{2} \\
& =K_{3} s^{|\boldsymbol{M}| / 2} s^{m}\left\|P(D) E *\left(\rho_{s}-\rho_{s / 2}\right)\right\|_{2}=K_{3} s^{|\boldsymbol{M}| / 2} s^{m}\left\|\lambda_{s}\right\|_{2} \\
& =K_{3} s^{|\boldsymbol{M}| / 2} s^{m} s^{-|\boldsymbol{M}| / 2}\left\|\lambda_{1}\right\|_{2}=K_{4} s^{m}
\end{aligned}
$$

где $K_{2}, K_{3}$ и $K_{4}$ не зависят от $s$. Отсюда и из неравенства Минковского вытекает, что последовательность $g_{2-l}(\boldsymbol{x}), l \in \mathbb{N}$, фундаментальна в $L_{1}(B)$. Следовательно, она сходится в $L_{1}(B)$ при $l \rightarrow \infty$ к некоторой функции из $L_{1}(B)$, которая, очевидно, совпадает в $B \backslash\{\boldsymbol{0}\}$ с $E(\boldsymbol{x})$. Таким образом, $E \in L_{1}\left(\mathbb{R}^{n} ;\right.$ loc $)$. Далее, при каждом $p \in[1, \infty]$ имеет место неравенство

$$
\|\Phi\|_{p} \leqslant K_{5} r^{m}\|P(D) \Phi\|_{p},
$$

где $\Phi$ - та же функция, что и в $(11)$, а $K_{5}$ зависит только от $P(D)$, которое в силу приведенных вьше соображений достаточно доказать в случае $\boldsymbol{x}=\boldsymbol{0}, r=1$. Используя обобщенное неравенство Минковского (см., например, [10, с. 27-28]), имеем

$$
\begin{aligned}
\|\Phi\|_{p} & \leqslant\|E * P(D) \Phi\|_{p} \leqslant\left(\int_{|\boldsymbol{x}|<1}\left(\int_{|\boldsymbol{x}-\boldsymbol{y}|<1}|(P(D) \Phi)(\boldsymbol{x}-\boldsymbol{y}) E(\boldsymbol{y})| d \boldsymbol{y}\right)^{p} d \boldsymbol{x}\right)^{1 / p} \\
& \leqslant\left(\int_{|\boldsymbol{x}|<1}\left(\int_{|\boldsymbol{y}|<2}|(P(D) \Phi)(\boldsymbol{x}-\boldsymbol{y}) E(\boldsymbol{y})| d \boldsymbol{y}\right)^{p} d \boldsymbol{x}\right)^{1 / p} \\
& \leqslant \int_{|\boldsymbol{y}|<2}|E(\boldsymbol{y})| d \boldsymbol{y} \cdot\|P(D) \Phi\|_{p}=K_{5}\|P(D) \Phi\|_{p}, \quad p<\infty
\end{aligned}
$$

Выше мы воспользовались тем, что при $|\boldsymbol{x}|<1$ из $|\boldsymbol{x}-\boldsymbol{y}|<1$ вытекает, что $|\boldsymbol{y}|<2$. Если $p=\infty$, то в цепочку неравенств (13) нужно внести соответствующие изменения. Теперь, как и при $p=2$, из (12) получаем, что для каждого $p \in[1, \infty]$ имеет место неравенство

$$
\left\|g_{s}-g_{s / 2}\right\|_{p} \leqslant K_{6} s^{m} s^{-|M| / q}\left\|\lambda_{1}\right\|_{p}
$$

где $K_{6}$ не зависит от $s, q=1+(p-1)^{-1}$. Как и вьше, проверяем, что в случае $m-|\boldsymbol{M}| q^{-1}>0$ последовательность функций $g_{2^{-l}}(\boldsymbol{x})$ сходится при $l \rightarrow \infty$ в $L_{p}(B)$ к $E(\boldsymbol{x})$, откуда сразу получаем необходимое утверждение.

Показатель $p$ в следствии 5 точен, на что указьвают известные примеры фундаментальных решений однородных эллиптических операторов (см. [4, с. 206]). Отметим, что следствие 5 может быть получено в качестве следствия результатов В. В. Грушина [11], относящихся к оценкам роста фундаментальных решений произвольных гипоэллиптических уравнений вблизи особенности.

ЗАмЕчАниЕ 2. В процессе доказательства следствия 5 получена оценка

$$
\left\|g_{s}-g_{s / 2}\right\|_{p} \leqslant K s^{m-|M| / q}\left\|\lambda_{1}\right\|_{p}
$$

где $1 \leqslant p \leqslant \infty, q=1+(p-1)^{-1}, K$ не зависит от $s$. 


\section{СПИСОК ЦИТИРОВАННОЙ ЛИТЕРАТУРЫ}

[1] Zalcman L. Mean values and differential equations // Israel J. Math. 1973. V. 14. P. 339-352.

[2] Fulks W. A mean value theorem for the heat equation // Proc. Amer. Math. Soc. 1966. V. 17. P. 6-11.

[3] Купцов Л. П. О свойстве среднего для уравнения теплопроводности // Матем. заметки. 1981. T. 29. № 2. C. 211-222.

[4] Хёрмандер Л. Анализ линейных дифференциальных уравнений с частными производными. Т. 1. М.: Мир, 1986.

[5] Хёрмандер Л. Анализ линейных дифференциальных уравнений с частными производными. Т. 2. М.: Мир, 1986.

[6] Хёрмандер Л. Введение в теорию функций нескольких комплексных переменных. М.: Мир, 1968.

[7] Ван дер Варден Б. Л. Алгебра. М.: Наука, 1979.

[8] Волевич Л.Р. Локальные свойства решений квазиэллиптических систем // Матем. сб. 1962. Т. 59 (101). Доп. вып. С. 3-52.

[9] Эдвардс Р. Функциональный анализ. Теория и приложения. М.: Мир, 1969.

[10] Никольский С. М. Приближение функций многих переменных и теоремы вложения. М.: Наука, 1977.

[11] Грушин В. В. Связь между локальными и глобальными свойствами решений гипоэллиптических уравнений с частными производными // Матем. сб. 1965. Т. 66 (108). № 4. С. 525-550.

Государственная академия легкой промышленности Украины, г. Киев

Поступило 30.12 .96 